\title{
Frequently Asked Questions on the mean field configuration interaction method. I- Distinguishable degrees of freedom.
}

\author{
P. Cassam-Chenaï, A. Ilmane \\ Laboratoire Dieudonné, UMR 6621 CNRS-UNS, Faculté des Sciences, Parc \\ Valrose, F-06108 Nice cedex 2, France.
}

\begin{abstract}
The mean field configuration interaction (MFCI) method has been developped in recent years both for electrons (EMFCI) and molecular vibrations (VMFCI). In the present article we provide complements and further details on this topics in the form of a "frequently asked questions" text. The first part focuses on the molecular vibrational problem.
\end{abstract}

Keywords:

mean field, configuration interaction, geminal and group functions, contracted vibrational modes.

Suggested running head: faq on MFCI

All correspondance to be send to P. Cassam-Chenaï, cassam@unice.fr, tel.: +33492076260, fax: +33493517974. 


\section{Introduction}

The mean field configuration interaction (MFCI) method has been developed originally by Cassam-Chenaï and Liévin for molecular vibrations and was called vibrational MFCI (VMFCI) [1]. It has proved extremely powerful and flexible to solve the molecular, vibrational, stationary Schrödinger equation [2-4]. This has encouraged one of the authors to develop a version of the MFCI method for fermions. The latter has been implemented for molecular electrons in the computer code TONTO and called the "electronic MFCI" (EMFCI) method [5-8]. In contrast with the VMFCI method implemented in the computer code CONVIV $[9,10]$ in full generality, the EMFCI method implemented in TONTO is restricted at present to the case where the electrons are grouped in pairs, that is to say, are represented by antisymmetrized products of geminals. This particular case of EMFCI is referred to as the geminal MFCI (GMFCI) method.

Both the VMFCI and the EMFCI have been presented in seminars and at international conferences, in particular at the "international meeting on Mathematical Methods for Ab Initio Quantum Chemistry" held in Nice, annually since 2005. We have gathered in this article, presented in the form of a "frequently asked questions" (FAQ) text, many of the questions rised during discussions having taken place at these occasions, or elsewhere. The purpose of the article is to clarify certain points addressed too succinctly in previous publications and to present some subtleties of the MFCI method, or of its computer implementation, never mentioned before. Some questions actually go beyond the mere scope of the MFCI method and touch upon problems inherent in variational methods, in general.

The article is organized as follows: We begin with a brief account of the VMFCI method, in order to make the article accessible to non-specialists. The EMFCI method is not explicitly formulated in this first part which focuses on the VMFCI method, however the main steps follow those of the VMFCI. Then, we begin the faq section itself. In one question, differences between the VMFCI and EMFCI methods and algorithms are outlined.

Part II of this work will be mainly devoted to the EMFCI method. 


\section{The VMFCI method explained}

The VMFCI method consists in performing vibrational configuration interactions of some degrees of freedom (DOFs) in the mean field of the other. The aim of the method is to keep the finite basis sets used in successive vibrational configuration interaction (VCI) within manageable sizes, by contracting groups of DOFs together, as in the traditional contraction method [11-14]. However, the power of the method comes from the mean field term added to the group Hamiltonians not present in the traditional approach.

Partitions of DOFs

A VMFCI step starts with a partition, $P$, of the $n_{v i b}$ vibrational degrees of freedom into $n_{P}$ subsets :

$$
P=\left(I_{1}, I_{2}, \cdots, I_{n_{P}}\right)=\left(\left\{i_{1}^{1}, i_{2}^{1}, \cdots, i_{k_{1}}^{1}\right\},\left\{i_{1}^{2}, i_{2}^{2}, \cdots, i_{k_{2}}^{2}\right\}, \cdots,\left\{i_{1}^{n_{P}}, i_{2}^{n_{P}}, \cdots, i_{k_{n_{P}}}^{n_{P}}\right\}\right)
$$

Using partition $P$ the vibrational Hamiltonian can be written as :

$$
\begin{aligned}
H_{v i b}=h_{0} & +\sum_{\gamma_{1}=1}^{n_{P}} h_{\gamma_{1}}\left(I_{\gamma_{1}}\right) \\
& +\sum_{\substack{1 \leq \gamma_{1}<\gamma_{2} \leq n_{P}\\
}} h_{\gamma_{1}, \gamma_{2}}\left(I_{\gamma_{1}}\right) h_{\gamma_{1}, \gamma_{2}}\left(I_{\gamma_{2}}\right) \\
& +\cdots+h_{1,2, \cdots, n_{P}}\left(I_{1}\right) h_{1,2, \cdots, n_{P}}\left(I_{2}\right) \cdots h_{1,2, \cdots, n_{P}}\left(I_{n_{P}}\right)
\end{aligned}
$$

where $h_{\gamma_{1}, \gamma_{2}, \ldots, \gamma_{k}}\left(I_{\gamma_{l}}\right)$ denotes a vibrational operator that only depends upon operators acting on DOFs in subsets $I_{\gamma_{l}}$.

Then, one defines a possibly coarser partition, $Q=\left(J_{1}, J_{2}, \cdots, J_{n_{Q}}\right)$, satisfying $n_{Q} \leq n_{P}$ and $\forall \gamma \in\left\{1, \cdots, n_{P}\right\}, \exists \alpha \in\left\{1, \cdots, n_{Q}\right\}$ such that $I_{\gamma} \subseteq J_{\alpha}$.

For such a step, we call "contractions" the subsets $J_{\alpha}$, and "components of contraction $J_{\alpha}$ " the subsets $I_{\gamma}$ such that $I_{\gamma} \subseteq J_{\alpha}$. When several VMFCI steps are performed, the components of one step are the contractions of the previous step. Note that the case $Q=P$ is allowed. Iterating the same partition until self-consistency lead to vibrational self-consistent field configuration interaction (VSCFCI) methods, which generalize the 
well-known VSCF method $[15,16]$. Such a generalization has been considered by Bowman and Gazdi [17] but not in the frame of an iterated VCI approach.

\section{Product basis sets}

Let us consider a given contraction $J_{\alpha}$ that we will call "active". The other contractions are called "spectators". We assume that contraction $J_{\alpha}$ has $\beta$ components :

$$
\begin{aligned}
J_{\alpha} & =I_{\gamma_{1}} \cup I_{\gamma_{2}} \cup \cdots \cup I_{\gamma_{\beta}} \\
& =\left\{i_{1}^{\gamma_{1}}, \cdots, i_{k_{\gamma_{1}}}^{\gamma_{1}}, \cdots, i_{1}^{\gamma_{\beta}}, \cdots, i_{k_{\gamma_{\beta}}}^{\gamma_{\beta}}\right\} \\
& =\left\{j_{1}^{\alpha}, \cdots, j_{l_{\alpha}}^{\alpha}\right\} \quad \text { with } \quad l_{\alpha}=k_{\gamma_{1}}+\cdots+k_{\gamma_{\beta}} .
\end{aligned}
$$

Having a basis set $\left\{\phi_{I_{\gamma}}^{m_{\gamma}}\right\}_{m_{\gamma} \in\left\{1, \ldots, d_{\gamma}\right\}}$, spanning an Hilbert subspace of dimension, say $d_{\gamma}$, for each component $I_{\gamma}$, we build for contraction $J_{\alpha}$, a so-called "product basis set", $\left\{\Phi_{J_{\alpha}}^{M_{\alpha}}\right\}_{M_{\alpha} \in\left\{1, \ldots, D_{\alpha}\right\}}$, spanning an Hilbert subspace of dimension $D_{\alpha}$, by constructing product functions of the form:

$$
\Phi_{J_{\alpha}}^{M_{\alpha}}=\bigotimes_{I_{\gamma} \subseteq J_{\alpha}} \phi_{I_{\gamma}}^{m_{\gamma}}
$$

or more explicitly, writing variable dependencies:

$$
\begin{aligned}
\Phi_{J_{\alpha}}^{M_{\alpha}}\left(q_{i_{1} \gamma_{1}}, \cdots, q_{i_{\gamma_{1}}}^{\gamma_{1}}, \cdots, q_{i_{1}^{\gamma_{\beta}}}, \cdots, q_{i_{k_{\beta}}^{\gamma_{\beta}}}\right) & =\Phi_{J_{\alpha}}^{M_{\alpha}}\left(q_{j_{1}^{\alpha}}, \cdots, q_{j_{l_{\alpha}}^{\alpha}}\right) \\
& =\prod_{I_{\gamma} \subseteq J_{\alpha}} \phi_{I_{\gamma}}^{m_{\gamma}}\left(q_{i_{1}^{\gamma}}, \cdots, q_{i_{k_{\gamma}}^{\gamma}}\right)
\end{aligned}
$$

with $\quad M_{\alpha}=\left(m_{\gamma_{1}}, \cdots, m_{\gamma_{\beta}}\right)$.

In CONVIV, the process is initialized with an basis set of modals, that is to say, functions of a single vibrational degree of freedom, but this constraint can be walked around if groups of DOFs are contracted from the start. The modal basis available at present in CONVIV either are built from eigenfunctions of one dimensional Schrödinger equations with various potential such as a harmonic potential (with arbitrary center and frequency), a Morse potential [18], a Trigonometric Pösch-Teller (TPT) potential [19], a Kratzer potential [20], or are Chebychev polynomials.

By convention, the eigenstates are numbered in increasing order with natural numbers starting with 0 , so the vector index, $M_{\alpha}=(0, \ldots, 0)$, always corresponds to product of 
ground state functions.

The dimension of the basis set for the contraction $J_{\alpha}$ can be different from the product of dimensions of its component's basis sets because of possible basis function truncations, usually performed according to some energy criteria (vide infra).

\section{Mean field Hamiltonian}

For the active contraction $J_{\alpha}$, we define a partial Hamiltonian, $H_{\alpha}$, by grouping all the terms in $H_{v i b}$ involving the DOFs in components $I_{\gamma_{l}}$ of $J_{\alpha}$ :

$$
\begin{aligned}
& H_{\alpha}=h_{0}+\sum_{\substack{\gamma_{1} \\
\text { such that } \\
I_{\gamma_{1}} \subseteq J_{\alpha}}} h_{\gamma_{1}}\left(I_{\gamma_{1}}\right)+\sum_{\begin{array}{c}
\gamma_{1}<\gamma_{2} \\
\text { such that } \\
I_{\gamma_{1}}, I_{\gamma_{2}} \subseteq J_{\alpha}
\end{array}} h_{\gamma_{1}, \gamma_{2}}\left(I_{\gamma_{1}}\right) h_{\gamma_{1}, \gamma_{2}}\left(I_{\gamma_{2}}\right) \\
& +\cdots+\sum_{\substack{\gamma_{1}<\cdots<\gamma_{\beta} \\
\text { such that } \\
I_{\gamma_{1}}, \cdots, I_{\gamma_{\beta}} \subseteq J_{\alpha}}} h_{\gamma_{1}, \cdots, \gamma_{\beta}}\left(I_{\gamma_{1}}\right) \cdots h_{\gamma_{1}, \cdots, \gamma_{\beta}}\left(I_{\gamma_{\beta}}\right)
\end{aligned}
$$

In contrast with the original contraction method, a mean field term accounting for the average effect of all the spectator modes, is added to this partial Hamiltonian:

$$
\widetilde{H_{\alpha}}=H_{\alpha}+\left\langle\bigotimes_{I_{\gamma} \nsubseteq J_{\alpha}} \phi_{I_{\gamma}}^{0}\left|H_{v i b}-H_{\alpha}\right| \underset{I_{\gamma} \nsubseteq J_{\alpha}}{\bigotimes} \phi_{I_{\gamma}}^{0}\right\rangle
$$

A VMFCI calculation consists in performing a VCI [21] for the mean field Hamiltonians, Eq.(3), in the product basis sets, Eq. (1). In CONVIV, at any one step, a VMFCI is performed for each contraction of the $Q$-partition.

Thereby, we obtain new basis sets $\left\{\phi_{J_{\alpha}}^{m_{\alpha}}\right\}_{m_{\alpha} \in\left\{1, \ldots, D_{\alpha}\right\}}$ of dimension $D_{\alpha}$ made of eigenvectors of the mean field Hamiltonians to construct the product basis sets of the next step. Their associated eigenvalues can be used to truncate the product basis sets according to energy criteria: either the individual component basis functions are selected if their associated eigenvalue is less than a given threshold, or a product function is selected if the sum of its component eigenvalues is less than a given threshold. Both criteria can be applied jointly.

Note that in CONVIV, it is possible to control the number of eigenpairs calculated by the diagonalizer when solving the mean field Hamiltonian eigenvalue problem. So, in 
fact, the dimension of the new basis set for contraction $J_{\alpha}$, can be less than $D_{\alpha}$.

Question 1: Could the VMFCI method, although termed "vibrational", be applied to the Hamiltonian of any system made of distinguishable degrees of freedom (DOFs), such as the molecular Hamiltonian of nuclear motion, including rotational and possibly translational DOFs?

Yes, in fact the VMFCI method, and the computer code CONVIV, can be applied to any set of distinguishable degrees of freedom (DOFs). Test calculations, yet unpublished, with a pseudo-rotation internal motion DOF or with rotational motion of the whole molecule DOFs coupled with the vibrational DOFs have, actually, already been performed with the code CONVIV.

Question 2: If the answer to question 1 is yes, why not employing the VMFCI method for the complete nuclear motion Hamiltonian in Cartesian coordinates, which assumes a particularly simple and universal form?

In principle traditional quantum mechanics does not depend upon its representation. So, it is tempting to use the simple Cartesian Hamiltonian. Test calculations can easily be carried out with CONVIV. However, the results are disastrous, even if a unitary transformation over the Cartesian DOFs is performed to make appear rectilinear vibrational, rotational and translational DOFs, as in the standard normal coordinate approach. Rectilinear rotational DOFs are absolutely not adapted to describe the proper rotational motion: the kinetic part of the Cartesian Hamiltonian is very different from that of a rigid rotator Hamiltonian, for example, and the potential expansion in terms of powers of these DOFs converges too poorly to produce a spectrum that resembles a rotational spectrum, when it is truncated. Furthermore, when using the MFCI method, the fact that the rotational DOFs are poorly described, affects the vibrational DOFs that are optimized in the mean field of the latter. 
Question 3: Then, can one retrieve the form of the exact (that is non truncated) potential involving the rectilinear rotational coordinates from the back-transformation of the Hamiltonian in the Eckart frame to the Cartesian Hamiltonian in the laboratory frame, and deal with this exact potential?

The problem is that, in this potential, the rectilinear rotational coordinates will be coupled to the other rectilinear coordinates in a non separable manner, (that is to say, it cannot be cast in the form of a finite sum of products of one-DOF factors). A priori, this will not favor an effective use of the MFCI approach.

Question 4: The variational principle insures that the energy can only decrease at each VMFCI calculation. This is provided, one performs a VMFCI calculation for a single active contraction per step. However, in CONVIV, one performs a VMFCI calculations for all the contractions of a given partition at the same step, using spectator ground states functions of the previous step. Is it to speed up the convergence?

The answer is yes, the variational principle is sacrified to speed up convergency in the CONVIV computer code. So far, we have noticed only one occurence of zero point energy increase instead of decrease in successive CONVIV calculation steps. As can be seen in Tab. 1, this increase was only by a small amount, and the convergence was again satisfactory at the next step.

However, the situation is different in the case of the EMFCI method, where it is often observed that after a few iterations, the increase of the ZPEs of different electron groups block the convergence of the calculation, when the EMFCI algorithm is implemented in the CONVIV way. This is because the optimized ground state $\Psi_{0}^{(1)}$ of, say electron group number 1 , affects the ground state of group $2, \Psi_{0}^{(2)}$, not only by the mean field it produces, but also by modifying its effective contribution to the antisymmetric product function of the total ground state, $\Psi_{0}^{(1)} \wedge \Psi_{0}^{(2)}$. (We use the "exterior" or "wedge product" notation [22].)

Let us consider a not very realistic, yet instructive, case example. Suppose that the exact 
wave function of a 4-electron system is, $\psi_{1} \wedge \bar{\psi}_{1} \wedge \frac{\psi_{2} \wedge \bar{\psi}_{3}+\psi_{3} \wedge \bar{\psi}_{2}}{\sqrt{2}}$, and that we start from an SCF guess of the form $\psi_{1} \wedge \bar{\psi}_{1} \wedge \psi_{2} \wedge \bar{\psi}_{2}$ in a GMFCI calculation. $\psi_{1}, \psi_{2}$ and $\psi_{3}$ are 3 orthonormal orbitals spanning our basis set. At step 0 , if one starts with group 1 active, using the mean field associated to $\psi_{2} \wedge \bar{\psi}_{2}$, one will find a solution of the form, $\Psi_{0}^{(1)}=a_{11} \psi_{1} \wedge \bar{\psi}_{1}+a_{33} \psi_{3} \wedge \bar{\psi}_{3}+a_{13} \frac{\psi_{1} \wedge \bar{\psi}_{3}+\psi_{3} \wedge \bar{\psi}_{1}}{\sqrt{2}}$, (geminal basis functions containing $\psi_{2}$ or $\bar{\psi}_{2}$, are not considered with such a spectator geminal). Whereas, if one starts with group 2 active, using the mean field associated to $\psi_{1} \wedge \bar{\psi}_{1}$, one will obtain, $\Psi_{0}^{(2)}=\frac{\psi_{2} \wedge \bar{\psi}_{3}+\psi_{3} \wedge \bar{\psi}_{2}}{\sqrt{2}}$. With the algorithm currently implemented in TONTO, both active groups are considered at step 0 . The one giving the lowest ground state energy is retained and its new ground state is combined with the old spectator ground state that has served to build the mean field Hamiltonian. So, the exact solution is found immediately, since it corresponds to the VMFCI calculation with group 2 active. With an algorithm à la CONVIV one would combine the new ground states of both groups:

$$
\begin{aligned}
& \left(a_{11} \psi_{1} \wedge \bar{\psi}_{1}+a_{33} \psi_{3} \wedge \bar{\psi}_{3}+a_{13} \frac{\psi_{1} \wedge \bar{\psi}_{3}+\psi_{3} \wedge \bar{\psi}_{1}}{\sqrt{2}}\right) \wedge \frac{\psi_{2} \wedge \bar{\psi}_{3}+\psi_{3} \wedge \bar{\psi}_{2}}{\sqrt{2}}= \\
& a_{11} \psi_{1} \wedge \bar{\psi}_{1} \wedge \frac{\psi_{2} \wedge \bar{\psi}_{3}+\psi_{3} \wedge \bar{\psi}_{2}}{\sqrt{2}}-a_{13} \psi_{3} \wedge \bar{\psi}_{3} \wedge \frac{\psi_{1} \wedge \bar{\psi}_{2}+\psi_{2} \wedge \bar{\psi}_{1}}{2},
\end{aligned}
$$

which is not a variational procedure and which does not give the exact solution.

This sort of negative "interference" between wave functions obtained by separate GMFCI calculations for different groups is not possible for a VMFCI wave function, because its tensor product components cannot mix in the same way. Still, the variational principle is lost if the mean field averaging is performed from step-(n-1), approximate, spectator, ground states for all contractions of the step-n partition, and this might result in a ZPE increase. However, as a matter of fact, this has never been an issue, when using CONVIV, so far.

\section{Question 5: Can one focus on a given spectral window with VMFCI?}

This is a general issue for any variational method. The answer is a priori no for a given Hamiltonian operator [24], because if there are holes in the lower part of the computed spectrum, the basis functions designed to describe the eigenfunctions of the spectral window of interest, may indeed serve to describe the missing eigenfunctions at the expense 
of the eigenfunctions of interest. An example of such a phenomenon will be provided in the answer to Question 10. To circumvent this limitation, one can think of filtering techniques, which consist in transforming the Hamiltonian, $H \rightarrow f(H)$, by applying a function, $f$, possibly parametrized by the energy bounds of the window of interest, which carries the selected levels at the bottom of the spectrum of the transformed Hamiltonian, (see Wyatt and coworkers for references and applications to vibrational spectroscopy $[25,26])$.

However, if the excited states of interest correspond to excitations of a given set, $S$, of DOFs, then the flexibility of the VMFCI method can be profitably used. One can for example avoid to contract the DOFs in $S$ with the DOFs not in $S$, and then truncate drastically the basis sets of contractions containing only DOFs not in $S$. This may produce holes in the spectrum, but in general the latter will not affect the excited states of interest. Of course, there should be no resonnance between DOFs in $S$ and DOFs not in $S$ below the targeted states.

\section{Question 6: Can one average over a different spectator state than the ground state to focus on a particular excited state?}

Yes, but only at the last step of a calculation with CONVIV. Otherwise, if one more iteration is performed, the new spectator states to be used in the computation of the mean field correction may not have the right physical meaning. So, they will not be suitable for the purpose of building mean field Hamiltonians. For example, let us consider a 3 DOF system. Suppose that the targeted state is the first excited state in DOF number 1. If, at step $n$, one averages the Hamiltonian for DOF number 3 over the first excited state of DOF number 1 and the ground state of DOF number 2, the new ground state so-obtained for DOF number 3 will not be a proper ground state for this DOF, because the true ground state would correspond to a ground state product mean field. Then, at step- $(n+1)$, if one uses this pseudo-ground state for DOF 3, to compute the mean field Hamiltonian for DOF 1 or DOF 2, an unphysical spectrum will result. 
Question 7: The CONVIV code permits to choose between many types of basis functions, and most types depend upon one or more free parameters. How to select the type of basis set and adjust the parameters?

It is important to note that there is no universal criterium to decide whether a basis set is better than another. For a 1-D problem, it seems natural to choose a basis type corresponding to a model potential that ressembles the exact one. For example, if the shape of the exact potential is close to that of a Morse potential, it is tempting to use Morse basis functions. Then, it is likely that, this choice will be close to optimal in terms of speed of convergence with respect to the number of basis functions. However, if the aim of the computation is not the best description of a large part of the lower spectrum with a finite basis set of as small a size as possible, but is, instead, a very accurate description of a few lower levels, this choice may not be appropriate, since there are only a finite number of bound states for the Morse potential. It might be more advantageous to opt for a basis set of harmonic functions with well-chosen center and frequency, for example. For small basis set sizes, arguably, it will be less accurate than the Morse basis of the same size. However, it will be possible, in principle, to add as many basis functions as required to achieve the accuracy goal. Other criteria, such as the computational cost of calculating Hamiltonian matrix elements could be considered.

Regarding the optimization of the free parameters, several strategies can be considered. One can minimize the difference between selected eigenvalues of the 1-D Hamiltonian corresponding to a 1-D section of the PES and those corresponding to the model potential. For example, if we consider that, the best frequency for a harmonic modal basis, is the one for which the largest number of eigenvalues are converged within the $\mathrm{cm}^{-1}$ accuracy given a fixed number of basis functions, one find that the best harmonic modal basis of 50 functions for a Morse potential with parameters, $D=0.181077$ au, $a=0.0242094161$ $\mathrm{au}$, has a frequency of about 0.4 times the harmonic frequency, $\nu_{0}$, of the Morse potential second order Taylor expansion [27]. More precisely, with an harmonic modal frequency equal to $\nu_{0}$, the first 10 levels are within the $\mathrm{cm}^{-1}$ accuracy of the exact Morse eigenvalues, whereas with a modal frequency of $0.4 \nu_{0}$, four more levels are converged.

Alternatively, one can optimize the parameters by fitting the PES to a model potential below an energy threshold corresponding to the spectral region of interest. Examples will 
be provided in the following questions. For a given energy threshold, say $\lambda$, fitting the curves on a large grid of points covering the energy region below $\lambda$, or minimizing the eigenvalues discrepancies below $\lambda$, can result in very different sets of parameters. Again, which parameters are the best, will depend on the aim of the calculation to be performed using the modal basis set.

\section{Question 8: Should one use 1-D sections of the original PES or mean field 1-D potential curves to optimize modal basis sets?}

The problem of choosing modal basis sets is more complicated for a multidimensional system, because the optimal modal basis for a 1-D section of the potential may not be optimal to describe the couplings with the other DOFs. In a VMFCI context, the mean field potential of a given DOF, which takes into account the average effect of the intermode couplings, is a priori preferable to the corresponding 1-D section of the PES, in view of solving the multidimensional problem. As a matter of fact, it has been shown that the anharmonicity due to inter-mode couplings, not included in 1-D section of the original potential, usually dominates the anharmonicity due to intra-mode couplings [28]. Furthermore, Table II of [3] shows that 1-D sections of the original potential give eigenvalues worse than those of a simple harmonic approximation in half of the cases, and worse than those obtained from mean field potentials in all cases.

Mean field potentials for modal basis optimizations depend upon the choice of spectator ground state basis functions. Harmonic basis functions corresponding to a quadratic approximation of the PES, though very simple, are in general good enough to provide qualitatively correct mean field 1-D potential without the need to perform a single VMFCI calculation. In a few cases, such as mode $\nu_{3}$ in Table II of [3], a better approximation of ground state spectator modes would be required. This could be the solution of a step 0 MFCI calculation, or, possibly, the ground state product of better model potentials for spectator DOFs.

However, a posteriori, the differences between the low-lying eigenvalues obtained from optimized modal basis with or without mean field, have been found unsignificant in many cases. For example, in Fig. 1, the differences between the two potential curves with and without mean field correction, though significant, are much smaller than the differences 
of model potentials in Fig. 3, which themselves have little influence on the lowest levels obtained from VCI calculations, as seen from Tab. 2.

Other interesting considerations regarding the optimization of modal functions for a multidimensional Hamiltonian can be found in [31].

\section{Question 9: How to optimize the modal basis set of an anisotropic degenerate mode?}

When the symmetry group of a molecule is non Abelian, some vibrational modes can be degenerate, say $d$-times degenerate, and the $d$-dimensional section of the PES, when the other DOFs are at the equilibrium geometry, can be anisotropic. This is the case for example of the $\nu_{2}$-mode of methane illustrated in Fig. 2 (upper panel).

However, to perform VMFCI calculations with finite modal basis that do not break molecular symmetry, the parameters of the modal basis must be the same in the directions of all the d-degenerate coordinates. So, it is a priori important to choose parameters that would give basis functions appropriate in any direction of the $d$-dimensional potential.

For the $\nu_{2}$-mode of methane, the influence of potential anisotropy on the optimal frequency of a harmonic basis set, is small enough to be negligible, see lower panel of Fig. 2. The optimal frequency was determined by mean square fitting the harmonic potential to the potential curve in direction $\theta$, (see Fig. 2, upper panel), on a grid of points below an energy of $22000 \mathrm{~cm}^{-1}$. The closer to equilibrium, the less the anisotropy, so one expects a larger effect at higher energy. However, even with a larger effect on the optimal frequency, it is expected, as in the previous question, that the optimal frequency variations will hardly affect the bottom of the spectrum. So, to answer the question, in practice, one can choose any direction in the degenerate subspace, and use the optimal frequency in that direction to construct the modal basis sets in all $d$-dimensions. 
Question 10: When the PES has non physical regions, as is often the case with polynomial expansion, can one play on basis set parameters to increase the number of basis functions having a significant weight only in the physical domain and improve the description of the bound states?

The PES used in molecular spectroscopy are seldom global ones, having correct asymptotic behaviors. They are often polynomial expansion, fitted on a grid of nuclear configuration points relatively close to the equilibrium geometry, or obtained by analytical or numerical derivatives of an electronic energy expression. In particular, commercial quantum chemistry codes now deliver quartic potential amenable to a second order perturbative treatment [33]. However, these PES can have spurrious oscillations, as one goes away from equilibrium, and may even be non bounded from below, which is an issue for variational methods.

To answer the question, let us consider the model quartic potential of Fig. $3, V(x)=$ $8 * 10^{-5} x^{2}-1.6 * 10^{-8} x^{4}$, (in au), whose shape is close to the 1 -D section of a $10^{\text {th }}$ order potential for methane [29], represented in Fig. 1. We assume that the non physical region starts beyond the barrier in both directions. We have calculated the bound levels of this potential by performing VCI calculations with three different harmonic modal basis corresponding to the harmonic potentials also represented on Fig. 3. The fitted harmonic potential has been obtained by mean square fitting a grid of points in the dotted region of the quartic potential.

Figure 4 displays for each modal basis, the first ten VCI eigenvalues for increasing numbers of basis functions. For all modal basis, the pattern is similar: The levels alternate decreasing phases with stabilizing phases. The first 9 levels stabilize first at the expected bound state eigenvalues. Then, the levels dive in cascade, that is one after the other, starting from the highest ones, before stabilizing again, except the two lowest ones, which appear to dive to minus infinity. If we extrapolate the "Fitted harmonic" case, for any level, say level $n$, there is an inflexion when it reaches level $n-1$, then a new stabilization at level $n-2$, and so on, with successive stabilizations at levels $n-2 k$, as long as, $k>\frac{n}{2}-1$. The highest $(n=10)$ level also stabilizes at level $n-2=8$, once the latter level has dropped.

According to the Hylleraas, Undheim, MacDonald theorem [34,35], the levels can only 
decrease as the number of basis functions increases. This is of course verified. However, this theorem tells nothing about the pace of the decrease, which is remarkable in the present case. The observed steps can be interpreted as follows: with a small number of basis functions, the modal basis is too incomplete to describe the high bounded levels, specially when the modal frequency has not been optimized: the further from the fitted frequency, the steepest are the initial slopes. Then, as the basis set is being completed, the bounded levels tend to converge (first stabilization area). However, increasing the basis set size eventually adds functions having non negligible weight in the unbounded region beyond the barrier. The description of the wave functions of the highest levels is affected first, since they have more overlap with the newly introduced basis functions. They tend to dive to $-\infty$, however, when a lower level has already dived, they stabilize to describe the missing bound level, provided it has the same parity.

So, when there are unbounded regions in the potential, adding more functions can spoil a variational calculation. One can think of using a stiff frequency to increase the number of basis functions having negligible weight in the unbounded region. As matter of fact, with the stiff harmonic potential of Fig. 3, 40 basis functions can be used before the $8^{\text {th }}$ level drops, against 26 for the fitted harmonic potential. Let us analyse the performance of these basis set with the help of Tab. 2 .

For a fixed, limited number of basis functions, columns " $n=15$ ", the fitted harmonic modal basis performs the best for all levels. This is somehow expected and justifies frequency optimization. When the number of functions reaches the instability area of the $8^{\text {th }}$ level, one sees that, for all modal basis, the first four levels have converged to the same values, and that levels 9 and 10 have both dropped in accordance with Fig. 4. Regarding levels 5 to 8, it is clear that the fitted harmonic modal basis, whose maximum number of basis functions is limited to 26, is inferior to the other basis. Level 5 has converged with both the standard harmonic basis sets (corresponding to the potential quadratic constant) and the stiff harmonic basis set. Levels 6 to 8 obtained with the 40 functions of the stiff harmonic basis are lower than those of the standard harmonic basis limited at 29 functions. So, the stiff harmonic modal basis, which is slower to converge the low-lying levels than the other basis, becomes interesting for the highest bounded levels. However, it is important to note that not all the digits of these levels are converged. For example, level 6 with 30 standard harmonic basis function is equal to 14088.745504 
$\mathrm{cm}^{-1}$, that is to say, it is lower than all results of Tab. 2. In particular, this shows that, for this level, a standard harmonic basis of 30 functions performs better than the stiff harmonic basis of 40 functions.

To conclude this question, we note that, for any modal basis, the number of basis functions has to be finely tuned to obtain a given level at optimal accuracy. For example, with the stiff harmonic modal basis set, one would have to use less than 40 functions to obtain an estimate of level 9, but more than 40 functions would be required to lower the value of level 6 below the value obtained with 30 standard harmonic basis functions. For a harmonic basis set (with exponential factor equal to $\exp \left(-1 / 2(Q / a)^{2}\right)$ ), one could think of using the relationship, $Q_{M}=\operatorname{sqrt}(2 * n+1) / a$, between the classical spatial extension, $Q_{M}$, and the quantum number, $n$, of the level, to determine the appropriate number of basis functions, given the location of the unphysical region to be avoided. However, to get truely optimal numbers, one need to draw figures, such as Fig. 4, to determine the stability area of each level. This is heavy work. So, in the end, the best treatment is usually to fix the unphysical part of the potential, as has been done, for example, in [36] for a quartic PES of Formic acid.

\section{ACKNOWLEDGEMENTS}

The authors acknowledge the JAD laboratory computer facilities (J.-M. Lacroix and J. Maurin), and Prof. D. Jayatilaka for stimulating discussions. The authors are grateful to Prof. J. Liévin for sharing many of his, yet unpublished, results with them. 


\section{References}

[1] P. Cassam-Chenaï and J. Liévin, Int. J. Quantum Chem. 93, 245-264 (2003).

[2] P. Cassam-Chenaï, J. Quant. Spectrosc. Radiat. Transfer 82, 251-277 (2003).

[3] D. Bégué, C. Pouchan, N. Gohaud, P. Cassam-Chenaï, J. Liévin, J. Chem. Phys 127, 164115-164124 (2007).

[4] P. Cassam-Chenaï, Yohann Scribano, Jacques Liévin, Chem. Phys. Lett. 466, 16 (2008).

[5] P. Cassam-Chenaï, J. Chem. Phys 124, 194109-194123 (2006).

[6] P. Cassam-Chenaï, G. Granucci Chem. Phys. Lett. 450, 151-155 (2007).

[7] P. Cassam-Chenaï, V. Rassolov, Chem. Phys. Lett. 487, 147-152 (2010).

[8] http://sourceforge.net/projects/tonto-chem/

[9] P. Cassam-Chenaï, J. Liévin, Journal of Computational Chemistry 27, 627-640 (2006).

[10] https://forge.oca.eu/trac/conviv

[11] C. L. Chen, B. Maessen, and M. Wolfsberg,J. Chem. Phys 83, 1795-1807 (1985).

[12] J. Tennyson, Comp. Phys. Rep. 4, 1 (1986).

[13] S. Carter and N. C. Handy, Comp. Phys. Rep. 5, 15 (1986).

[14] Z. Bačić and J. C. Light, Annu. Rev. Phys. Chem. 40, 469 (1989).

[15] G. D. Carney, L. L. Sprandel and C. W. Kern in Advances in Chemical Physics, Vol. 37, I. Prigogine and S.A. Rice, Eds., (Wiley, New York, 1978), pp. 305-379.

[16] J. M. Bowman, J. Chem. Phys 68, 608-610 (1978).

[17] J. M. Bowman, B. Gazdy, J. Chem. Phys 94, 454-460 (1991).

[18] P. Morse, Phys. Rev. 34, 57-64 (1929).

[19] G. Pöschl, E. Z. Teller, Z. Physik, 83 (1933) 143

[20] A. Kratzer, Z. Physik 3,(1920) 289

[21] J. M. Bowman, K. Christoffel, F. Tobin,J. Phys. Chem. 83, 905-912 (1979).

[22] P. Cassam-Chenaï, F. Patras, J. Math. Phys. 44, 4884-4906 (2003).

[23] T. J. Lee, J. M. L. Martin and P. R. Taylor, J. Chem. Phys 102, 254 (1995). 
[24] J. Chang, N. Moiseyev, R. E. Wyatt, J. Chem. Phys 84, 4997 (1986).

[25] R. E. Wyatt, Phys. Rev. E51, 3643 (1995).

[26] T. J. Minehardt, J. D. Adcock, R. E. Wyatt, Phys. Rev. E56, 4837 (1997).

[27] J. Liévin, private communication.

[28] P. Cassam-Chenaï , F. Pauzat, et Y. Ellinger, J. Mol. Struct. (Theochem) 330, 167 (1995).

[29] A. V. Nikitin, M. Rey and Vl. G. Tyuterev, Chem. Phys. Lett. 501, 179 (2011).

[30] P. Cassam-Chenaï, Y. Bouret, M. Rey, S. A. Tashkun, A. V. Nikitin and Vl. G. Tyuterev, Int. J. Quantum Chem., in press (2011).

[31] R. Dawes and T. Carrington, J. Chem. Phys 122, 134101 (2005).

[32] D. L. Gray, A. G. Robiette, Mol. Phys. 1901, 37 (1979).

[33] V. Barone, J. Chem. Phys 122, 14108 (2005).

[34] E. A. Hylleraas and B. Undheim, Z. Phys. 657591930

[35] J. K. L. MacDonald, Phys. Rev. 438301933

[36] P. Cassam-Chenaï, J. Liévin, to be published. The original quartic PES of $\mathrm{CHOOH}$ was going to $-\infty$ beyond a barrier of only $600 \mathrm{~cm}^{-1}$ in the $\left(Q_{1}, Q_{9}\right)$-plane. To improve the PES, a grid of ab initio points in the range $Q 9=+/-64 \mathrm{au}, Q 1=+/-25$ au was constructed and fitted to an $8^{\text {th }}$ order polynomial. Other common methods, such as morsification of normal coordinates, were not found satisfactory. 


\section{Tables}

\begin{tabular}{|c|c|c|c|c|}
\hline \multirow[b]{2}{*}{ step type } & \multicolumn{4}{|c|}{$\mathbf{Z P E}$ in $\mathbf{c m}^{-1}$} \\
\hline & $\nu_{1}(10 \mathrm{bf})$ & $\nu_{3}(220 \mathrm{bf})$ & $\nu_{2}(120 \mathrm{bf})$ & $\nu_{4}(680 \mathrm{bf})$ \\
\hline MSP-VMFCI & $\mathbf{9 7 3 6 . 8 1 7 7 9 7}$ & $\mathbf{9 8 1 7 . 8 3 2 4 9 6}$ & $\mathbf{9 8 3 0 . 2 1 3 8 5 9}$ & $\mathbf{9 8 2 8 . 8 2 9 9 5 3}$ \\
\hline MSP-VMFCI & $\mathbf{9 7 2} 4.937053$ & $\mathbf{9 7 2 1 . 8 0 8 5 4}$ & $\mathbf{9 7 2} 4.971052$ & $\mathbf{9 7 2 4 . 6 2 4 9 5 2}$ \\
\hline MSP-VMFCI & 9721.492681 & $\mathbf{9 7 2 1 . 6 1 4 3 9 3}$ & $\mathbf{9 7 2 1 . 6 1 7 0 2 3}$ & $\mathbf{9 7 2 1 . 6 1 3 3 6 1}$ \\
\hline MSP-VMFCI & $\mathbf{9 7 2 1 . 4 9 4 0 1 6}$ & $\mathbf{9 7 2 1 . 4 8 9 7 4 7}$ & $\mathbf{9 7 2 1 . 4 9 4 2 3 1}$ & $\mathbf{9 7 2 1 . 4 9 3 7 5 9}$ \\
\hline MSP-VMFCI & $\mathbf{9 7 2 1 . 4 8 9 3 3 5}$ & $\mathbf{9 7 2 1 . 4 8 9 5 0 4}$ & $\mathbf{9 7 2 1 . 4 8 9 5 1 5}$ & $\mathbf{9 7 2 1 . 4 8 9 5 0 8}$ \\
\hline MSP-VMFCI & 9721.489334 & 9721.489328 & $\mathbf{9 7 2 1 . 4 8 9 3 3 5}$ & 9721.489334 \\
\hline MSP-VMFCI & 9721.489327 & 9721.489327 & 9721.489327 & 9721.489327 \\
\hline $\operatorname{VMFCI}\left(\nu_{1}-\nu_{3} ; 48000\right)$ & \multicolumn{2}{|c|}{$\mathbf{9 7 0 4 . 7 1 6 9 7 0}(1781$ bf $)$} & $\mathbf{9 7 2 1 . 4 8 9 3 2 7}$ & $\mathbf{9 7 2 1 . 4 8 9 3 2 7}$ \\
\hline $\operatorname{VMFCI}\left(\nu_{1}-\nu_{3} ; 22000\right)$ & 9704.716 & $70(330 \mathrm{bf})$ & $\mathbf{9 7 0 4 . 7 1 6 8 6 7}$ & $\mathbf{9 7 0 4 . 7 1 6 5 9 0}$ \\
\hline VCI(18349) & \multicolumn{4}{|c|}{9698.841643 (52096 bf) } \\
\hline
\end{tabular}

Table 1

ZPE convergence for a VMFCI contraction-truncation scheme in ${ }^{12} \mathrm{CH} 4$. At step 0 , the initial harmonic oscillator (HO) product basis set had 179,520,000 functions. MSP stands for minimal symmetry preserving, and correspond to the partitioning of the 9 DOFs into the 4 spectroscopic modes, $\nu_{1}, \nu_{2}, \nu_{3}, \nu_{4}$ of degeneracy $1,2,3,3$ respectively. This partition is used from step 0 to 6 . Then, at steps 7 and 8 , the two stretching modes, $\nu_{1}$, and $\nu_{3}$, are contracted together with truncation threshold on the sum of the product basis function energy at $48000 \mathrm{~cm}^{-1}$ and $22000 \mathrm{~cm}^{-1}$, respectively. This amounts to 1781 (resp. 330) basis functions (bf) in the VMFCI calculation of the stretching contraction (4 DOFs). Finally, in the last step, all DOFs are contracted in a VCI step. The converged digits for a given, iterated, partition are in bold. The ZPE decreases for all contractions of all partitions, except for mode 1 at step 3, where the ZPE slightly increases before decreasing again to even lower than the ZPE of the other modes. Such an increase is a very rare phenomenon in VMFCI calculations from our numerical experience. The tabulated numbers were obtained with the transformed potential energy surface of Lee, Martin and Taylor, [23], used in previous studies [1,2,9]. 


\begin{tabular}{|c|c|c|c|c|c|}
\hline \multicolumn{2}{|c|}{ Harmonic } & \multicolumn{2}{c|}{ Harmonic-Fitted } & \multicolumn{2}{c|}{ Harmonic-Stiff } \\
$(\omega=2776$ & $\left.c m^{-1}\right)$ & $(\omega=2438$ & $\left.c m^{-1}\right)$ & $\left(\omega=3709 c^{-1}\right)$ \\
\hline$n=15$ & $n_{\max }=29$ & $n=15$ & $n_{\max }=26$ & $n=15$ & $n_{\max }=40$ \\
\hline 1371.131862 & 1371.131862 & 1371.131862 & 1371.131862 & 1371.131862 & 1371.131862 \\
4077.968125 & 4077.968125 & 4077.968125 & 4077.968125 & 4077.968242 & 4077.968125 \\
6710.960673 & 6710.960668 & 6710.960669 & 6710.960668 & 6710.962240 & 6710.960668 \\
9263.366323 & 9263.365816 & 9263.365887 & 9263.365816 & 9263.451570 & 9263.365816 \\
11726.528807 & 11726.523475 & 11726.524298 & 11726.523476 & 11727.046640 & 11726.523475 \\
14088.962265 & 14088.745990 & 14088.778374 & 14088.745601 & 14099.134388 & 14088.745557 \\
16334.382810 & 16332.923383 & 16333.286920 & 16332.930070 & 16369.339290 & 16332.921818 \\
18454.111780 & 18429.000686 & 18438.080138 & 18426.096817 & 18701.752450 & 18422.067618 \\
20419.858316 & $\mathbf{1 9 8 6 7 . 9 6 4 6 8 5}$ & 20363.938015 & $\mathbf{1 9 3 3 6 . 4 4 5 0 0 1}$ & 20929.191041 & $\mathbf{1 8 8 7 0 . 6 3 7 5 0 2}$ \\
$\mathbf{2 2 4 7 2 . 2 5 1 3 1 7}$ & $\mathbf{2 0 4 4 3 . 2 4 9 1 3 2}$ & $\mathbf{2 2 1 9 8 . 8 3 3 7 2 4}$ & $\mathbf{2 0 1 6 3 . 3 1 2 8 3 8}$ & $\mathbf{2 3 9 7 2 . 5 4 0 2 7 9}$ & $\mathbf{1 9 6 8 4 . 4 8 2 4 7 4}$ \\
\hline
\end{tabular}

Table 2

First levels (in $\mathrm{cm}^{-1}$ ) of the model potential of Fig. 3 calculated with different basis sets. The value $n=15$ is situated in the middle of the stability region for all types of basis set. The $n_{\max }$ value represents the maximum number of basis functions before the instability of the $8^{\text {th }}$ level appears. More digits than significant physically are provided to appreciate numerical convergency. Bold numbers correspond to non stabilized eigenvalues according to Fig. 4. The digits that differ from those of the lowest number of each line are italicized. 


\section{Figures}

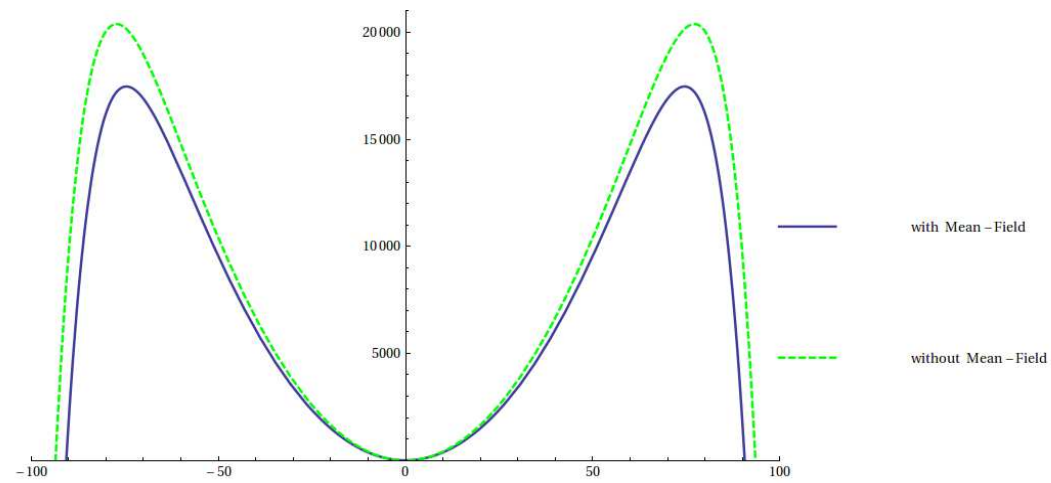

Fig. 1. $Q_{4 z}$-section of Nikitin, Rey, Tyuterev PES [29] transformed to mass-weighted normal coordinates [30] with and without mean field correction. The differences are much smaller than those between the harmonic potentials of Fig. 3. The basis sets obtained from the latter have, however, little influence on low-lying eigenvalues. Energy in $\mathrm{cm}^{-1}, Q_{4 z}$ in au. The mean field correction was obtained from harmonic approximations of spectator ground states. 

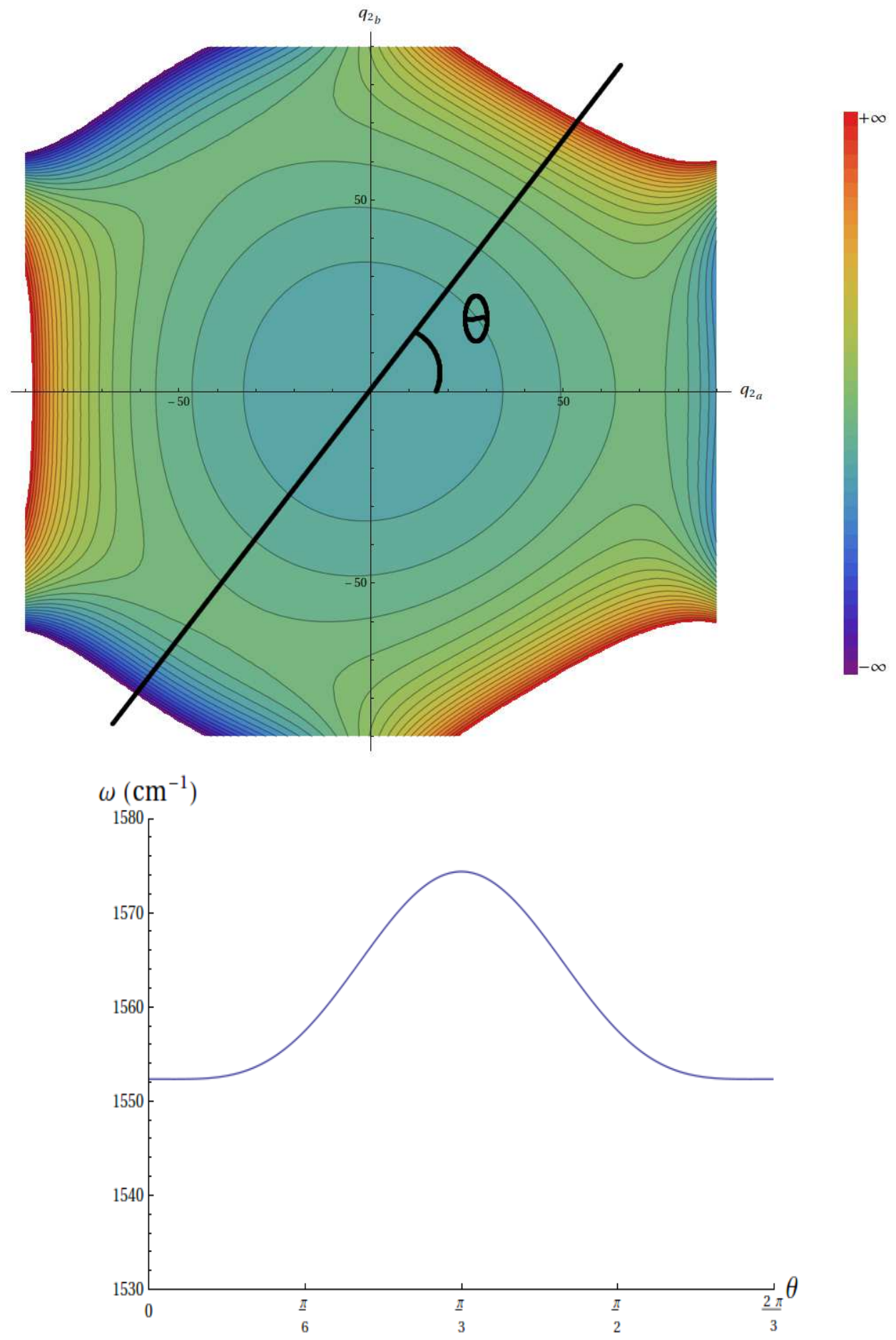

Fig. 2. Optimized harmonic modal basis set wave number as a function of angle $\theta$, for the 2-D section corresponding to the bending mode $\nu_{2}$, of the same methane PES as that used for Fig. 1. The sign convention for the mass-weighted normal coordinates $q_{2 a}$ and $q_{2 b}$ are those of Gray and Robiette [32]. The wave number has been optimized by mean-square fitting of a grid of 23400 points below $22000 \mathrm{~cm}^{-1}$, which corresponds to coordinate values falling in the interval $-60 \mathrm{au},+57 \mathrm{au}$. The effect of the anisotropy on the optimized wave number is not important enough to influence significantly the quality of the modal basis set. 


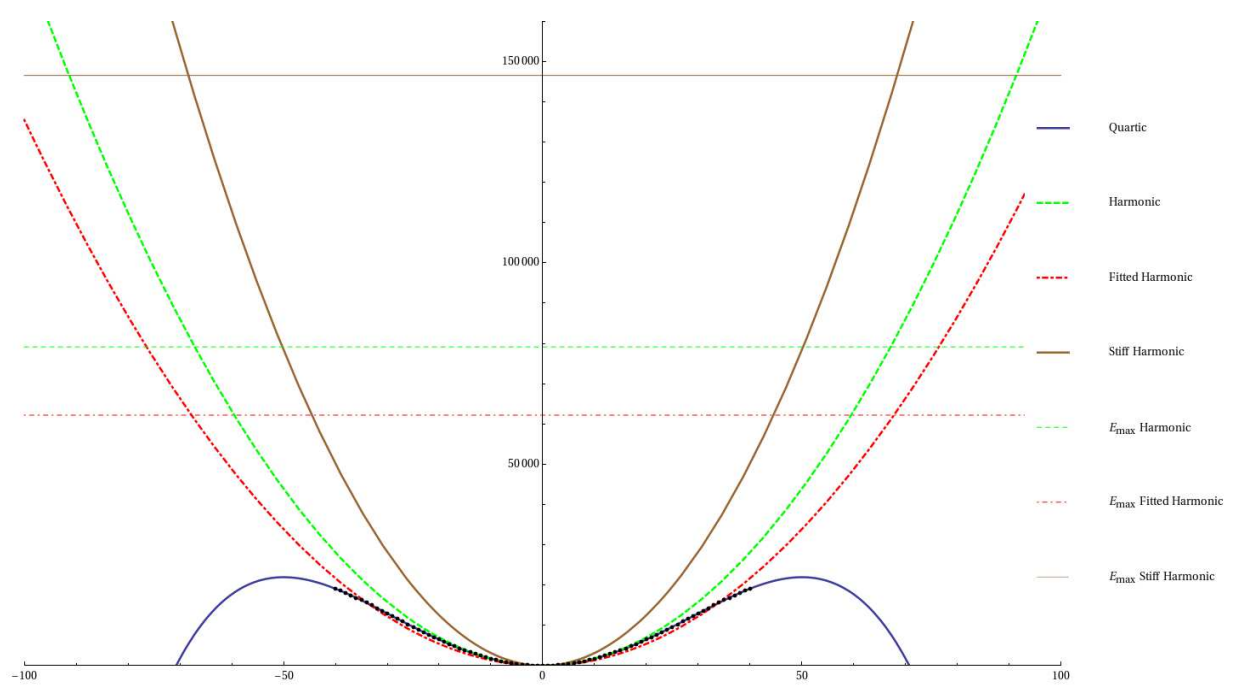

Fig. 3. Case-example of a quartic potential unbounded from below and model harmonic potentials whose eigenfunctions are used as basis functions. For each model potential, a horizontal line represents the energy level of the highest basis function before the $8^{\text {th }}$ bounded level of the quartic potential drops in Fig. 4. This highest basis function corresponds to the $n_{\max }$ value of Tab. 2. The barrier height of the quartic potential is about $21947.463 \mathrm{~cm}^{-1}$. The fundamental wave numbers of the harmonic, model potential are given in Tab. 2. This picture gives an idea of how rapidly the different basis sets extend beyond the barrier. 

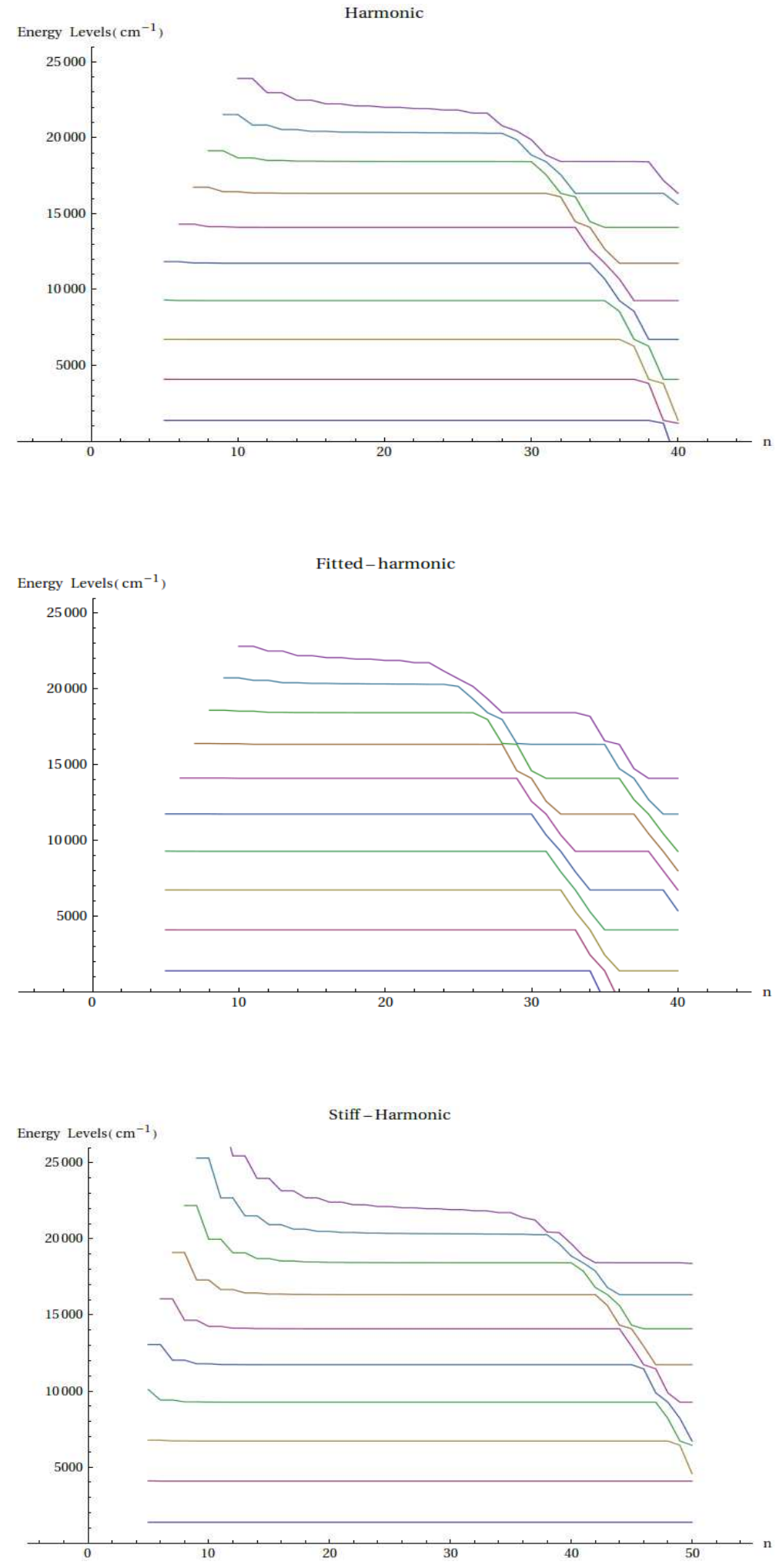

Fig. 4. VCI energy levels of the quartic potential of Fig. 3, as functions of basis set sizes. The basis functions are the eigenfunctions for the model potentials of Fig. 3. 